\title{
Circadian rhythm and pain: a modeling using multiagent systems
}

\author{
Angélica T. dos Santos ${ }^{1}$, Catia M. S. Machado ${ }^{1}$, Diana F. Adamatti ${ }^{1}$ \\ ${ }^{1}$ Programa de Pós Graduação em Modelagem Computacional (PPGMC) \\ Universidade Federal do Rio Grande (FURG) \\ Caixa Postal 474 - 96.203.900 - Rio Grande - RS - Brasil \\ \{theisangelica, catiamachado.furg, dianaada\}@gmail.com
}

\begin{abstract}
The circadian rhythm is responsible for the daily routines without metabolism and its disorders have great repercussion, such as obesity and mental disorders. This study is, the work approach has metate the study of synchronization and synchronization cycle circadian and influenced the variable of the, in the perspective of an integrated system with an computational system and computational. Results show the circadian rhythm periodicity is modified by the pain variable.
\end{abstract}

Resumo. O ritmo circadiano é responsável pelas variações diárias no metabolismo e seus distúrbios tem implicações diretas com muitas doenças, como a obesidade e transtornos mentais. Nesse sentido, o trabalho proposto tem como meta o estudo da sincronização e dessincronização ritmo circadiano influenciado pela variável da dor, sob a perspectiva de um sistema multiagente envolvendo um modelo matemático e computacional. Resultados mostram o quanto a periodicidade do ritmo circadiano é alterada pela variável dor.

\section{Introdução}

Os ritmos biológicos do ritmo circadiano são mudanças cíclicas que se repetem ao longo de um determinado período e estão relacionados com às alterações dos processos fisiológicos do corpo. Sua regulação é realizada por ritmos circadianos e homeostáticos [Borbely and Achermann 1999], onde é caracterizado pela redução significativa da atividade motora e da percepção de estímulos sensoriais.

Os modelos matemáticos são úteis para representar situações reais, para fazer previsões e auxiliar no apoio de decisões. Já os sistemas multiagente, área pertencente a inteligência artificial, permitem através de suas ferramentas, simular regras de comportamento de um determinado sistema, aproximando do fato real. Segundo [So and Durfee 1996], os agentes são personagens que atuam direto ou indiretamente, pelo usuário, podendo, os mesmos, atuar sozinhos ou em comunidades, formando um sistema multiagente.

A simulação é uma técnica que envolve a construção de um modelo de uma situação real para posterior experimentação. Em pesquisas de [Borbely et al. 1984], foi desenvolvido um modelo matemático que descreve as curvas do ritmo circadiano, sendo que [Borbely and Achermann 1999] aprimoraram este modelo, na qual [Skeldon 2014], implementou o modelo aprimorado utilizando sistema multiagente. 
Table 1. Escala Dor

\begin{tabular}{|c|c|c|c|c|c|}
\hline 0 & 2 & 4 & 6 & 8 & 10 \\
\hline Sem dor & Pouca dor & Dor Ligeira & Dor Moderada & Dor Intensa & Dor Máxima \\
\hline
\end{tabular}

Mediante isso, a contribuição deste trabalho é implementar a variável dor no modelo desenvolvido por [Skeldon 2014], seguindo o mesmo modelo matemático de [Borbely and Achermann 1999]. Desta forma, o objetivo geral é estudar o desempenho do ritmo circadiano influenciado pela variável dor, utilizando sistemas multiagente.

O texto está estruturado em 5 seções. Na seção 2 é apresentada a fundamentação teórica. Na seção 3 o modelo proposto por Skeldon, seguido da implementação realizada. Na seção 4 os resultados são apresentados e por fim, na seção 5 apresenta a conclusão.

\section{Referencial Teórico}

Os ritmos biológicos constituem-se do sistema de temporização endógeno. Esse sistema compreende a rede de osciladores acoplados, os quais, medem os ciclos ambientais claroescuro de 24 horas. Sabe-se que os ciclos ambientais não possuem a mesma capacidade de ajustamento, como a temperatura corporal varia em condições de rotina constante.

\subsection{Características do Ciclo Vigília-Sono}

O ciclo vigília-sono tem ritmicidade de 24 horas, em que o período de vigília ocorre durante o dia e o sono durante a noite, dando ênfase que o sono realizado durante o dia não tem a mesma qualidade do sono noturno, bem como a vigília que ocorre a noite não é igual à do dia. Assim, tem-se que o Processo $\tilde{\mathrm{S}}$ é união do ritmo circadiano e ritmo homeostático.

Um dos mecanismos importantes da regulação do vigília-sono, é mostrado pelo processo $\tilde{\mathrm{S}}$. Este por sua vez, é dependente da duração e qualidade do sono [Borbely and Achermann 1999]. A duração da vigília incrementa o processo $\tilde{S}$, aumentando assim o tempo de dormir. O vigília-sono é regulado pelo modelo de dois processos (ritmo circadiano e homeostático), definidos por [Borbely et al. 1984].

Segundo a Associação Internacional de Estudos da Dor (International Association for the Study of Pain - IASP), a dor é conceituada como "uma experiencia sensorial, emocional e desagradável, associada à um dano causado no corpo" [Chapman et al. 1985].

A dor é medida através da Escala Visual Analógica (EVA), que foi adaptada conforme a tabela 1. Na simulação multiagente, essa mesma tabela, foi usada para medir os níveis de dor. A dor influencia diretamente a qualidade do sono, sendo que a mesma pode provocar a dessincronização do ritmo circadiano.

\subsection{Ritmo Circadiano e Homeostático}

O ritmo circadiano, regula os ritmos materiais e psicológicos do ser humano, sendo controlado por um marca-passo localizado no cérebro, que é independente da vigília e do sono, que pode ser expresso por uma curva sinusoidal, com valores alcançando seu nível máximo de propagação do sono no início da manha e o seu mínimo no início da noite.

O ritmo homeostático é decorrente da vigília sono que procede do modelo $\tilde{\mathrm{S}}$, na qual é a pressão decorrente do sono acumulado durante o dia e que diminui durante a noite. 
O ritmo homeostático tem um aumento sinusoidal desde o início da vigília até o início do sono, na qual sofre uma queda até o seu final. O NREM é apresentado por marcadores fisiológicos do rito homeostático. O NREM é alto na primeiro estágio, quando é o primeiro sono, e exibe um declive sinusoidal ao longo do período [Borbely and Achermann 1999].

\subsection{Modelo Matemático}

O relógio biológico de cada pessoa é sincronizado conforme suas atividades decorrentes do dia. Na regulação interna, é necessário ter os mecanismos de ajustes que permitem a sincronização. Essa sincronização é realizada pelo fenômeno de ajuste, chamado de "arrastamento". Esse fator externo que comanda o ajuste, denomina-se "zeitgeber". Os "zeitgeber" são os sincronizadores do relógio biológico. O ritmo circadiano e homeostático são sincronizados pelo "zeitgebers", de maneira que os mesmos estejam sempre interligados, para que possam estar interligados é necessário um "pacemaker" (marcapasso). Para ocorrer a sincronização e dessincronização entre os ritmos, sempre tem um modelo matemático que comanda os ritmos [Borbely et al. 1984].

Conforme pode-se analisar pela equação (1), o ritmo homeostático.

$$
S_{t}=\left\{\begin{array}{c}
\left.d S_{t-1} ; d=\frac{-\Delta t}{\tau d}(\text { sleep })\right) \\
1-r\left(1-S_{t-1}\right) ; r=\frac{-\Delta t}{\tau d}(\text { wake })
\end{array}\right.
$$

Sendo que:

- $S$ : processo homeostático, que aumenta durante a vigília e diminui durante o sono;

- $d$ : fator de decaimento de $\mathbf{S}$;

- $r$ : fator de aumento de $\mathrm{S}$;

- $\tau d, \tau r$ constantes do tempo ;

- $\delta t$ tempo.

Nota-se que $\mathrm{S}$ é descrito por uma interação, onde o tempo são as etapas indicadas pelos índices t, t-1.

Ritmo Circadiano, equação (2).

$$
C=A\left\{\begin{array}{c}
0.97 \sin \left[\omega\left(t-t_{0}\right)\right]+0.22 \sin \left[2 \omega\left(t-t_{0}\right)\right]+0.07 \sin \left[3 \omega\left(t-t_{0}\right)\right]+ \\
0.03 \sin \left[4 \omega\left(t-t_{0}\right)\right]+0.001 \sin \left[5 \omega\left(t-t_{0}\right)\right] \\
\omega=\frac{2 \pi}{\tau}
\end{array}\right.
$$

Sendo que:

- $C$ : processo circadiano, que é independente da vigília sono;

- A: amplitude da onda sinusoidal;

- $t$ : tempo;

- $\tau$ período de $\mathrm{C}$;

- $t_{0}$ fase circadiana com o inicio da simulação.

Todos os parâmetros aqui apresentados, foram definidos por [Borbely et al. 1984], com base em dados obtidos a partir de adultos saudáveis, que foram submetidos a programas de vigília sono natural ou mudados. Estes parâmetros variam nos diversos tipos de simulação. 


\subsection{Sistemas Multiagente}

Uma importante modelagem de aprendizagem que vem sendo usada é a simulação multiagente. Nesse meio é possível mostrar uma população real em uma forma artificial, onde que cada indivíduo da população é apresentado por um agente e todos os agentes formam um grupo, sendo que cada um possui suas regras e comportamento.

A escolha pela simulação para a modelagem do ritmo circadiano se deu pela necessidade da inserção de diferentes comportamentos para os agentes, que facilita a modelagem, a interação entre os agentes, entre ambiente e agentes, além da flexibilidade das modificações e aplicações.

Há muitos ambientes de programação para modelagem baseada em agentes, dentre eles o NetLogo, que será utilizado nesse trabalho para simular o modelo do ritmo circadiano. O NetLogo é um ambiente de programação de modelagem, com intuito de analisar o comportamento dos fenômenos maturais. O mesmo utiliza a linguagem LOGO, com programação simples, sendo facilmente utilizada por pesquisadores de diversas áreas [Azevedo 2007].

\section{Modelo Proposto}

Nesta seção apresenta-se o modelo do ritmo circadiano baseado em agentes, que foi realizado a implementação por [Skeldon 2014]. Também apresenta a implementação que foi desenvolvida inserindo a variável da dor no modelo.

\subsection{Modelo do Ritmo Circadiano e Homeostático implementado por Skeldon}

O modelo que serviu de base para o estudo, parte dos pressupostos de [Skeldon 2014], onde é utilizada a simulação computacional, por meio do Netlogo. As variáveis iniciais que definem o modelo, são mostradas na figura 1, essas variáveis foram definidas por meio de dados empíricos. Cada variável foi inicialmente definida com valores, para que ao longo da programação, seja apenas necessário indicar a variável, sem precisar todas as vezes dizer o valor correspondente a mesma. A implementação das equações (1) e (2), mostradas na seção 2.3 é implícito no código em Netlogo.

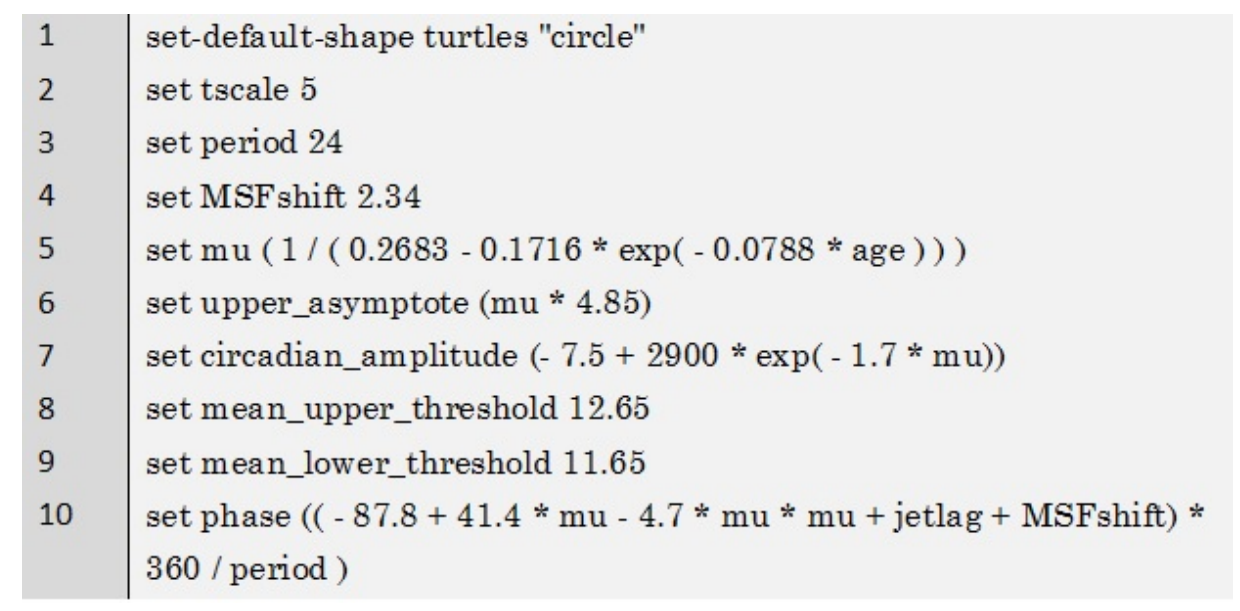

Figure 1. Variáveis do modelo matemático obtidas por meio de dados empíricos

A interface interativa do programa permite determinar variáveis, como, apresentadas na figura 2 . 


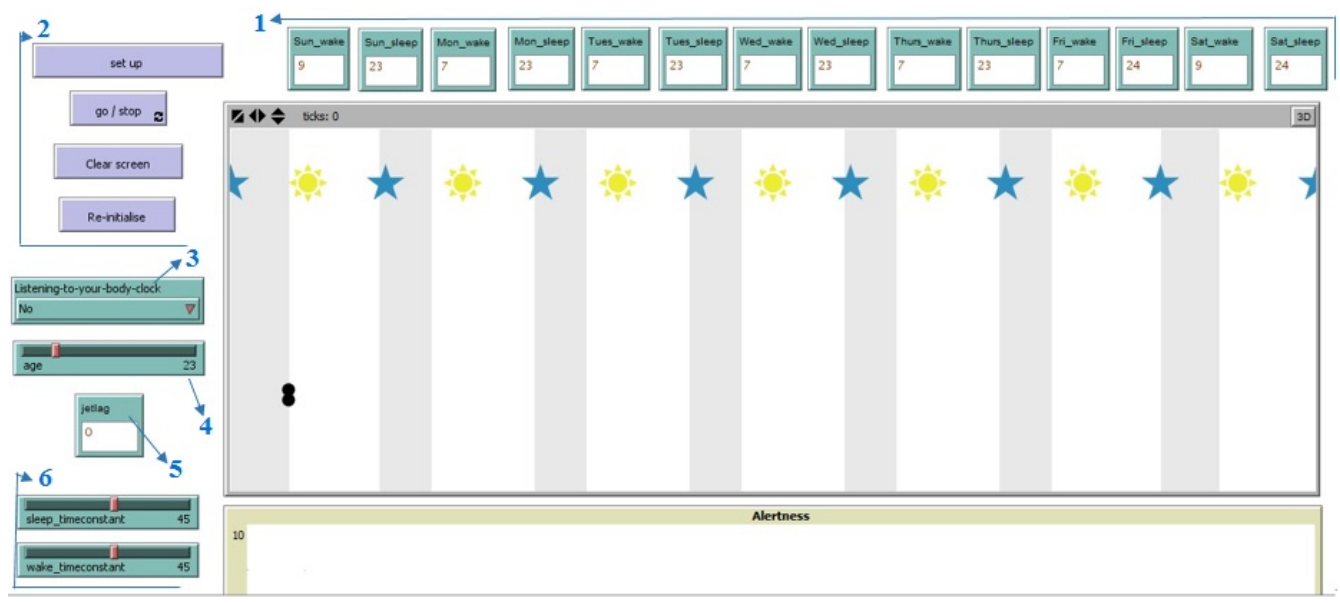

Figure 2. Interface do Modelo do Ritmo Circadiano

- 1 - Wake e Sleep - O horário de dormir e acordar.

- Set-up - Inicializar as informações da simulação; Go - Executar o programa; Reinitialise - Reiniciar o programa. Ticks - Tempo de execução do programa.

- 3 - Listening-to-your-body-clock: Yes ou No - Relógio Biológico: Ligado ou Desligado.

- 4 - Age - Idade.

- 5 - Jet-Lag - Fuso Horário.

- 6 - Wake-timeconstat e o Sleep-timeconstat - Tempo necessário para adormecer e acordar.

Desta maneira, analisa-se a figura 3, onde mostra o ritmo circadiano de um indivíduo por 7 dias. Percebe-se alguns fatores explicativos na figura:

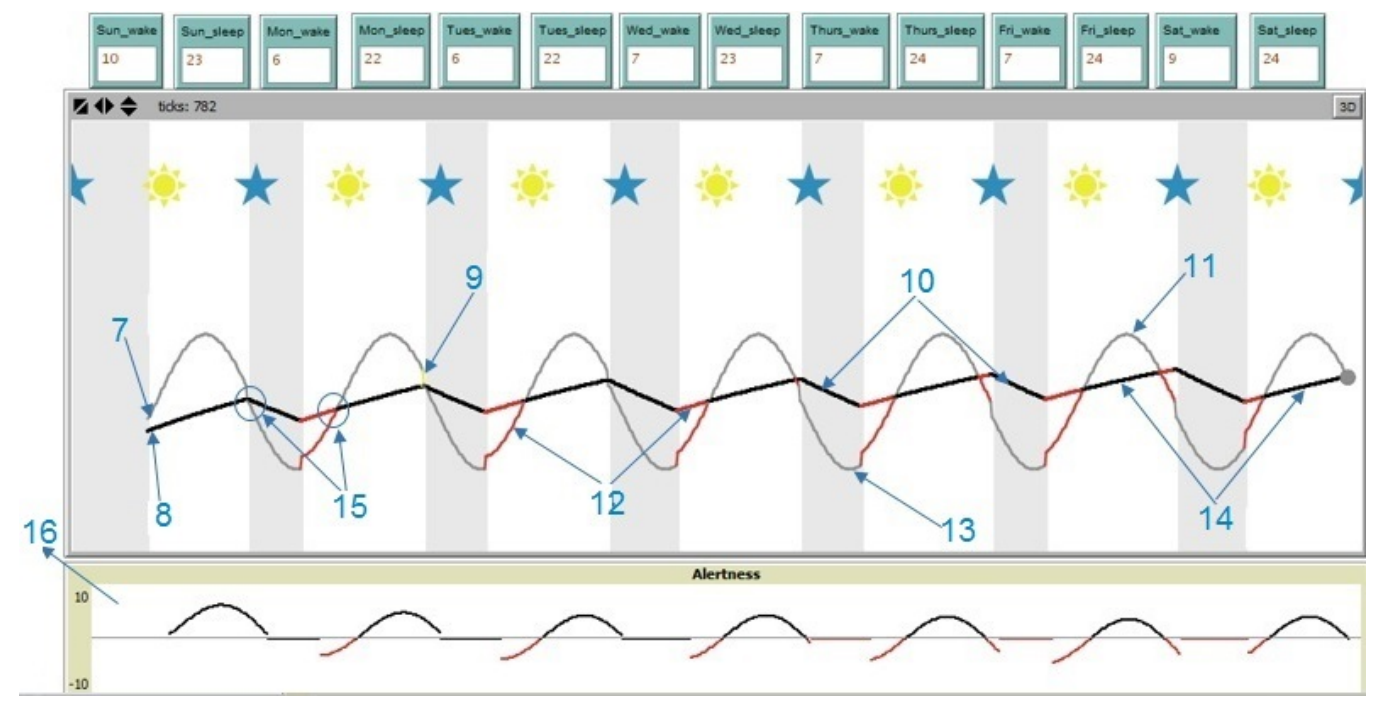

Figure 3. Interface do Modelo do Ritmo Circadiano 2

- O modelo mostra o ritmo homeostático e o ritmo circadiano que modulam e causam mudanças, da mesma forma que evoluem com o tempo em uma sequência de 7 dias. Para maior clareza, apenas o limiar superior é mostrado durante a vigília e o limiar inferior durante o sono. 
- 7 - Ritmo Circadiano - curva cinza

- 8 - Ritmo Homeostático - curva preta.

- 9 - As partes amarelas correspondem às vezes em que o relógio do corpo está lhe dizendo que deve estar acordado e, ainda assim, está tentando dormir com dificuldade.

- 10 - sleep - Dormir. Refere-se ao período da noite, momento em que o sono se realiza e o indivíduo adormece.

- 11 - Quanto mais tempo o indivíduo permanecer acordado, maior será a pressão do sono. No momento em que estamos ouvindo o ritmo biológico e indo dormir quando sentimos sono, ficamos adormecidos e a pressão do sono atinge o valor mais alto, chamado de limiar superior.

- 12 - As partes vermelhas correspondem às vezes em que o relógio do corpo está dizendo que a pessoa realmente quer estar dormindo, mas está acordada, refere-se ao sentimento de cansaço do dia a dia.

- 13 - Limiar inferior. O sono depende do quão longe o limiar superior atinge. Durante o sono, a sua pressão diminui até atingir um limiar mais baixo, momento em que acordamos, chamado de limiar inferior. Os limiares superior e inferior são modulados por um ciclo de 24 horas.

- 14 - Wake - Acordar. É o período do dia, momento de estar acordada, desenvolvendo as atividades do dia a dia, com sua eficiência máxima.

- 15 - Processo S̃. União do Ritmo Circadiano (7) e Ritmo Homeostático (8).

- 16 - Alertness - Alerta. Gráfico que mostra os períodos de alerta, momentos em que o indivíduo deveria estar dormindo.

Visualmente, a figura 3 detalha o ritmo circadiano de um indivíduo de 23 anos, que mantém seu ritmo quase controlado, sendo que nem todos os dias o mesmo dorme as 8 horas diárias recomendadas, isso sem levar em consideração o Wake-timeconstat e o Sleep-timeconstat, que é o tempo médio que o corpo leva para adormecer e acordar. As partes em vermelho surgem mais no período diurno, na parte da manhã, onde o mesmo queria estar dormindo, mas está acordado, pois o comando vindo do núcleo supraquiasmático do cérebro diz que o ritmo circadiano está em momento de descanso. Percebe-se que mesmo este indivíduo dormindo $8 \mathrm{~h}$ ou mais, seu corpo continua cansado, isso é influenciado pela sua idade.

\subsection{Modelo TAM}

Partindo do modelo base proposto por [Skeldon 2014], que analisa o Processo S̃ , união do ritmo circadiano e homeostático, o Modelo TAM tem seu diferencial pela inserção da variável dor, sendo a contribuição do trabalho.

A dor é uma variável que afeta a qualidade do sono, sendo que quando a pessoa tem dor a mesma pode não dormir, ou até dormir, mas não exerce o sono ideal para ter um descanso merecido.

Mediante, vários estudos realizados, procurou-se uma outra maneira de analisar a influencia da dor no ritmo circadiano. Assim, considerando todo o modelo matemático das equações (1) e (2) e a figura 1 que mostram parte do código, a dor é calculada inversamente proporcional, pela equação (3), que foi obtida por meio de testes com dados empíricos, onde encontrou-se o valor de 0.2955 , utilizando valores entre 0 e 1 , com uma ou mais casas decimais. 
Quanto mais próximo à 1 o valor, mais similar ao modelo de Skeldon situavase. Assim, foi analisado que o valor precisava ser baixo, quanto mais perto de 0.3 , mais mostrava os limiares do ritmo circadiano influenciado pela variável da dor. Desta forma, por meio do processo de testes chegou-se ao valor de 0.2955 , para ser o parâmetro de calibragem. ${ }^{1}$

$$
\text { pain } \rightarrow\left(1-\left(\frac{\text { pain }}{10}\right) * 0.2955\right)
$$

Somente com a equação (3) não é possível realizar o cálculo da dor. Para ser realizado o cálculo, a mesma necessita ser inserida no código em Netlogo, sempre levando em consideração a tabela 1 da escala da dor.

A variável da dor é inserida na "turtle 1 is the circadian" mostrado na figura 4. No código, a turtle 1 realiza a análise do horário de acordar, intensidade da dor, horário de dormir e intensidade da dor. O sistema multiagente, capta cada variável, inspeciona e realiza o cálculo da dor. Desta maneira o agente, a "turtle", percorre todo o dia, analisando a dor em relação ao período diurno e noturno.

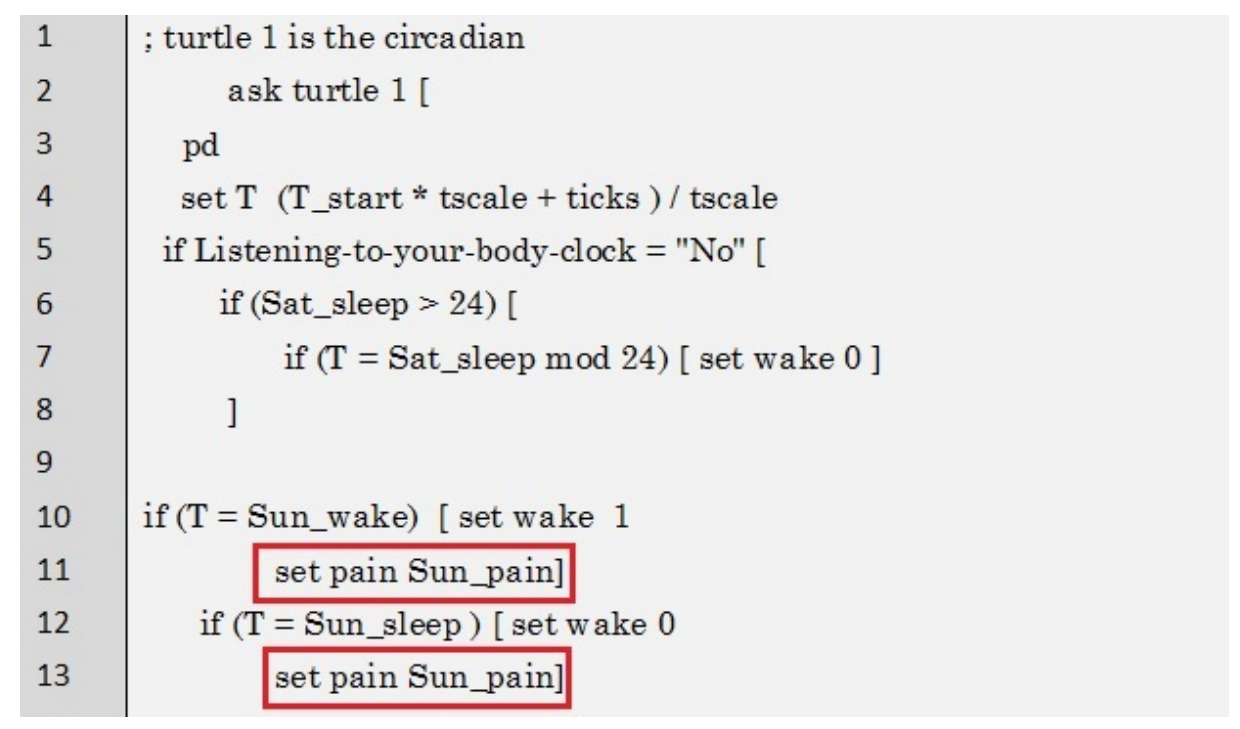

Figure 4. Influência da dor nos dias da semana

Desta forma, o agente apenas percorreu o circadiano para verificar os horários de acordar e dormir, não realizou o cálculo da intensidade da dor. $\mathrm{Na}$ figura 5, o agente realiza o cálculo do $H$ level, nível da pressão do sono que está acumulada no corpo, influenciada pela intensidade da dor.

O sistema multiagente percorre toda a semana, faz a análise de cada dia, baseado nos horários de dormir e acordar, e finalmente realiza o cálculo da dor por meio do $H$ level. O $H$ level é a variável que mostra a altura máxima e mínimo dos picos de dor, relacionado com a pressão do sono, o limiar superior e inferior.

\footnotetext{
${ }^{1} \mathrm{O}$ valor 0.2955 foi adequado através de testes empíricos.
} 


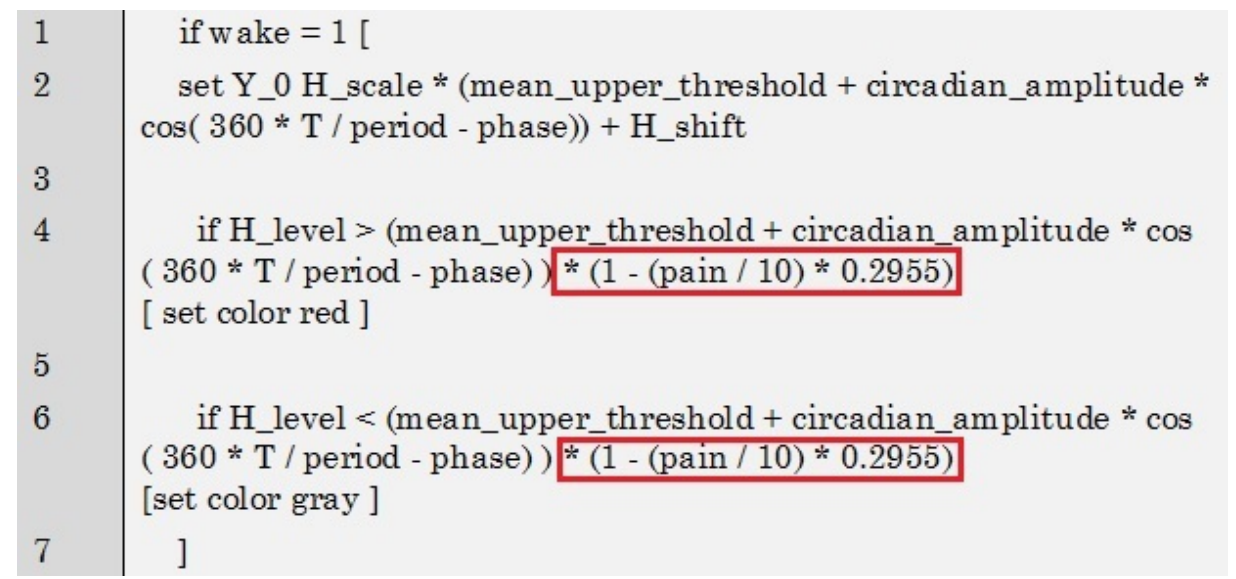

Figure 5. Cálculo da dor baseada nos parâmetros do ritmo circadiano

A EVA é definida através da tabela 1, adaptada com base os estudos de [Chapman et al. 1985] que mostra os níveis de dor, relacionado ao valor. O indivíduo que usar o programa deve dizer/inserir qual seu nível de dor, tendo como base a tabela 1.

A interface inicial do Netlogo, está definida, conforme a figura 6, onde o retângulo vermelho mostra a variável da dor.

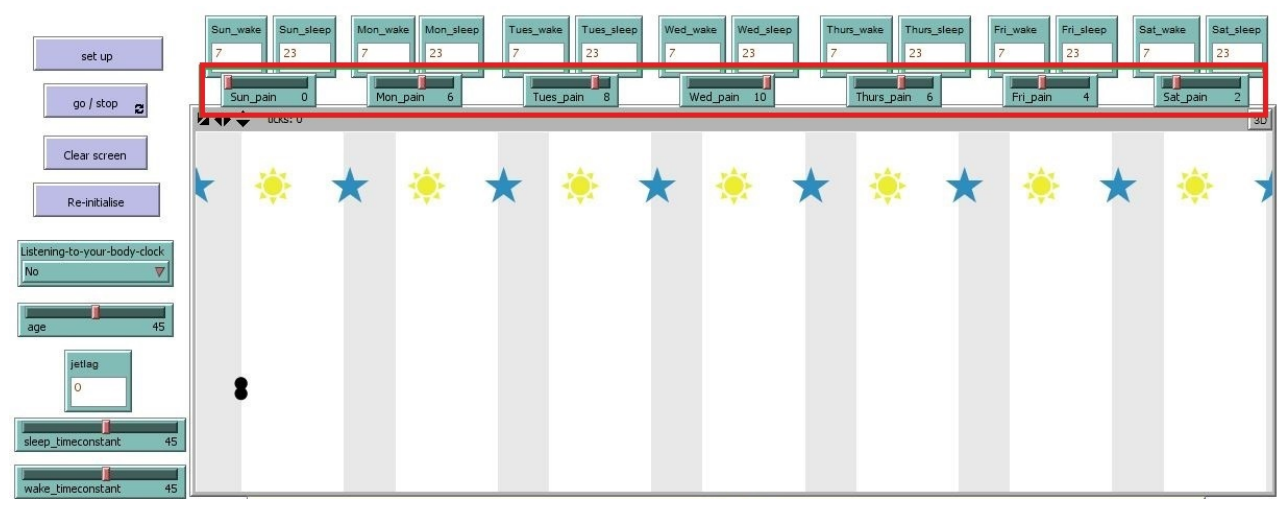

Figure 6. Interface final com a influência da dor

\section{Análise dos Resultados}

Os resultados foram obtidos por meio de testes, onde toda a análise é por meio de cenários, fazendo assim, uma comparação em relação à influência da intensidade do nível da dor.

Trocar o dia pela noite, é a opção que muitas pessoas tem, a mesma passa a dormir de dia, quando se trabalha à noite. Assim, muitos ritmos biológicos podem ficar comprometidos, modificando-se instantaneamente, levando a dessincronização interna. Essa dessincronização ocorre, quando a pessoa tenta dormir de dia, mas se sente alerta, que na verdade, ela precisa descansar no momento em que o seu corpo está preparado para a vigília [Simoneau et al. 1985].

Avante, a dessincronização interna está relacionado com o ambiente em que se realiza o sono de descanso, dor, a temperatura corporal, os hormônios, o estresse e as habilidades motoras, desânimo, fraqueza, insônia, sensação de "ressaca", descontrole, 
irritabilidade, agressividade, ansiedade, tremor, alterações gastro-intestinais, obesidade e envelhecimento precoce. Todos os fatores externos e internos prejudicam o sono.

É importante ressaltar, que para a idade, o wake timeconstant, o sleep timeconstant, jet lag, o horário de acordar e dormir, foram definidos valores padrões, para que desta forma, possa ser realizada uma análise mais detalhada referente à influência da intensidade da dor.

- Idade - 45;

- Wake e Sleep timeconstant - 45;

- Jet lag - 0;

- Horário de acordar - 7 horas;

- Horário de dormir - 22 horas;

- Cenário 1 - Influência do nível de dor zero;

- Cenário 2 - Influência do nível de dor quatro;

- Cenário 3 - Influência do nível de dor oito;

- Cenário 4 - Influência do nível de dor variável;

A escolha do indivíduo foi de forma aleatória, sendo que com 45 anos, a pessoa tem um sono controlado e quase sempre dorme as oito horas recomendadas.

Neste caso, é necessário cuidar que está definido sendo nove horas o período noturno de descanso. Destas nove horas é preciso levar em consideração o wake timeconstant, o sleep timeconstant, que é o tempo médio que o indivíduo leva para adormecer profundamente e acordar.

A taxa de "ticks" para todos os cenários de observação, está baseando entre setecentos e noventa a oitocentos e dez "ticks", sendo que nesse intervalo é possível realizar toda a simulação computacional para o período de sete dias.

A primeira observação realizada é mostrada na figura 7, onde o Relógio Biológico - Listening-to-your-body-clock estava desligado. Quando o mesmo encontra-se desligado, é necessário completar todas as informações, sendo que a influência do nível de dor zero mantém os ritmos do corpo em perfeita sincronização, pois o individuo não apresenta dor.

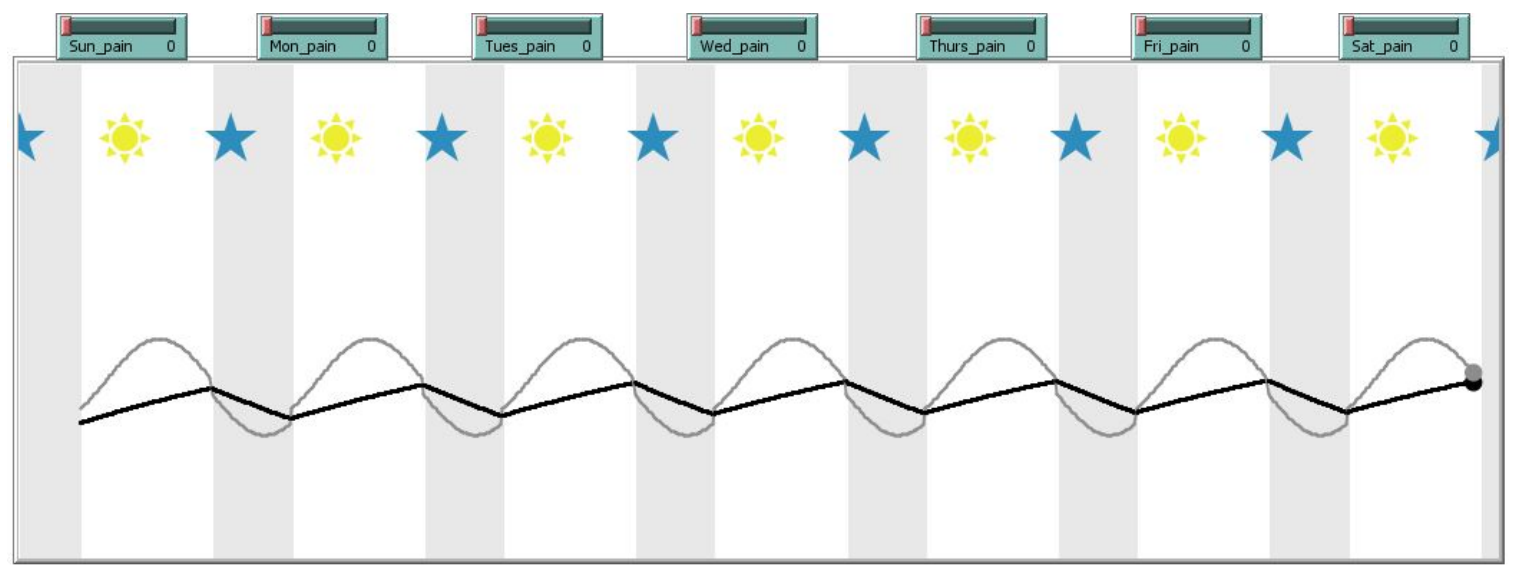

Figure 7. Cenário 1 - Influência do nível de dor zero

As curvas em cinza são mais acentuadas, sendo que após o encontro da curva cinza com a preta, a mesma passa a ter uma leve inclinação na horizontal, tanto no inicio 
da noite, quanto no inicio do dia. Esse encontro das curvas é o Processo S̃, união do ritmo circadiano e homeostático, sendo que quando os dois se encontram, suas curvas se cruzam e formam a sincronização perfeita deste indivíduo sem dor.

$\mathrm{Na}$ dor de nível quatro, figura 8, o indivíduo percebe que existe um incomodo presente em seu corpo, assim, modificando a sua percepção dos afazeres. Neste nível de dor, o indivíduo consegue realizar um sono adequado, com momentos de sono profundo e sono leve, seguido de alterações no nível do sono.

A dor apenas aparece no início do dia e da noite, quando os ritmos circadiano e homeostático se cruzam. Esses picos de dor, apresentam-se mais no final da semana, sendo sexta-feira e sábado, pois a mesma também leva em consideração os aspectos de cansaço e estresse do cotidiano.

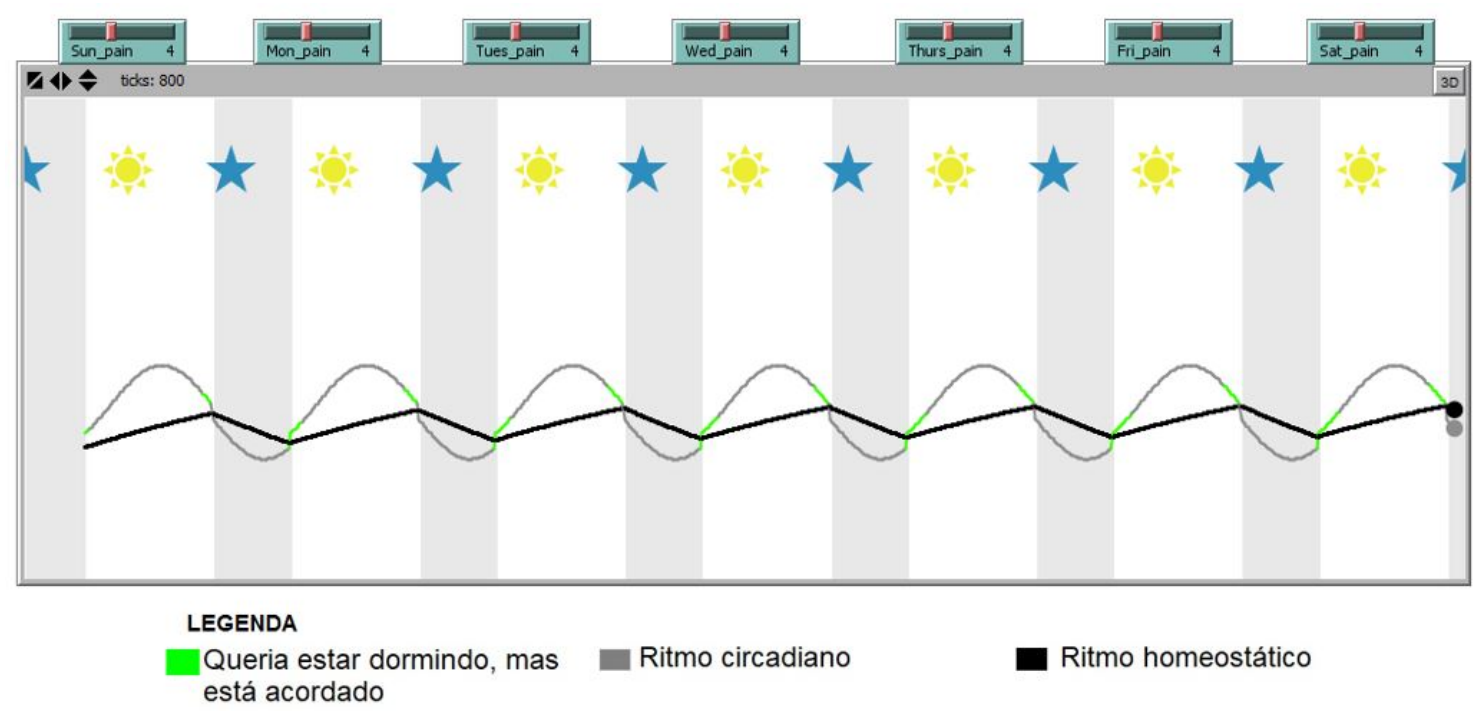

Figure 8. Cenário 2 - Influência do nível de dor quatro

Nem sempre o nível de dor está relacionado com alguma doença física, emocional ou psicológica. Quanto mais forte for o nível de dor, maior será os limiares em verde que aparecem na curva que mostra o período diurno.

As partes verdes correspondem às vezes em que o relógio do corpo está dizendo que a pessoa realmente quer estar dormindo, mas está acordada, refere-se ao sentimento de cansaço do dia a dia.

Quando a dor for mais intensa, de nível oito, a figura 9 propõe-se que os picos sejam elevados devido a intensidade da dor. O nível de dor oito, faz com que o indivíduo não desenvolva suas atividades corretamente, assim, consequentemente acarreta todo o seu psicológico. Acredita-se que o mesmo tem cochilos ao longo do período de 24 horas, pois é difícil ficar tanto tempo sem dormir. Neste caso, estamos desconsiderando o uso de medicação para dor.

A dor influência diretamente na qualidade do sono, sendo que quando o indivíduo tem dor, ele não tem o descanso merecido durante todos os dias.

Na maioria das vezes, o indivíduo não possui sempre a mesma intensidade do nível de dor $(2,6,10,8,6,4,2)$. Conforme a figura 10, o indivíduo teve níveis de dor 


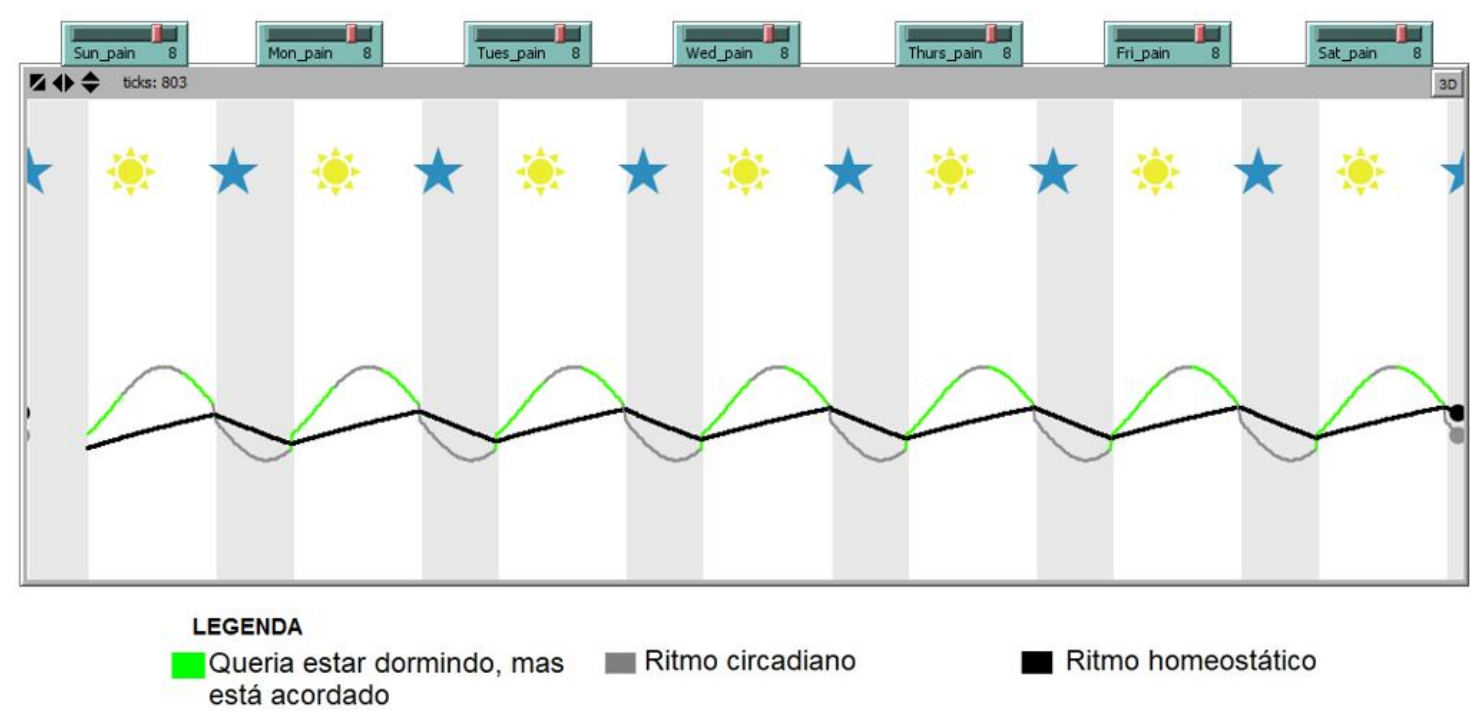

Figure 9. Cenário 3 - Influência do nível de dor oito

diferentes.

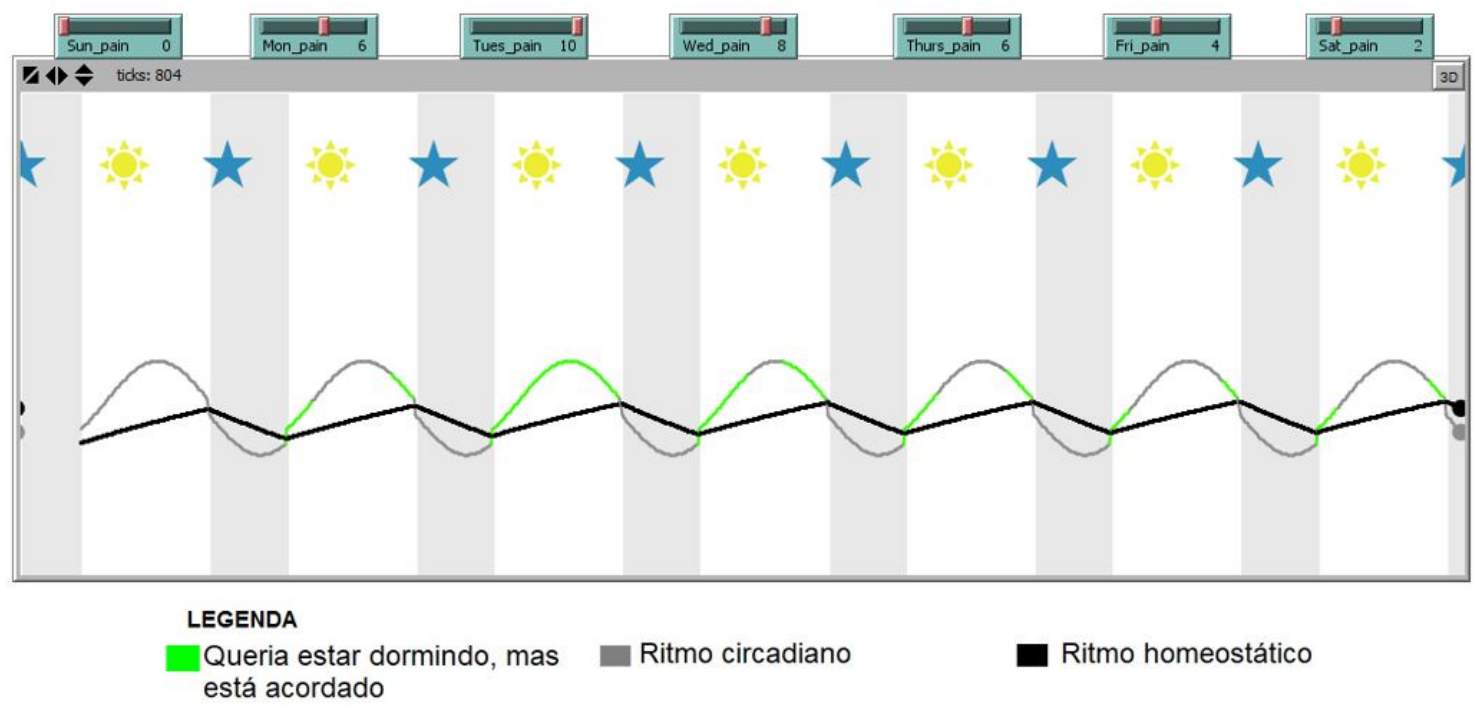

Figure 10. Cenário 4 - Influência do nível de dor variável

Com essa variação da influência dos níveis de dor, é perceptível, que como na terça-feira ele teve dor nível dez, no dia seguinte quarta-feira a dor diminuiu de nível, mais ainda continuou com um pico elevado de dor. Consequentemente, na dor aleatória, é intuitivo que o mesmo tenha toda a sua semana de observação alterada, sendo que após a dor dez, todos os dias ficaram comprometidos, pois é difícil ter dor dez em um dia e no seguinte não ter nada de dor.

Em relação as diferentes simulações, com varição do nível de dor, ainda pode-se realizar outros testes, levando em conta que pode-se variar a idade, tempo constante de dormir e acordar, o fuso horário. Todos estes resultados não podem ser comparados com dados reais, por não ter nenhum dado real, baseado em indivíduo com influencia do nível da dor. 


\section{Conclusões}

Este modelo apresentado por [Skeldon 2014] e [Borbely and Achermann 1999] garante de forma fidedigna a modelagem do ritmo circadiano, visto que todos os dados foram obtidos de adultos saudáveis, em programas de vigília do sono natural ou deslocados. Estes sujeitos foram expostos a vários reforços em seus ambientes sociais que ajudam o processo do sono-vigília a permanecer em sincronia com outros ritmos circadianos [Borbely et al. 1984]. Assim garante-se que variável da dor influência diretamente no ritmo circadiano.

Avaliando o comportamento do ritmo circadiano influenciado pela variável dor, utilizando sistemas multiagente, através das variáveis de simulação foi possível mostrar uma aplicação do dia a dia, onde o indivíduo sofreu alterações no nível de dor, durante o período de sete dias.

Outro ponto importante à salientar é que para o modelo matemático e o modelo computacional não havia uma publicação que explicasse como tudo foi desenvolvido. Assim, essa descoberta da interligação entre o modelo matemático e o modelo computacional, também é uma contribuição que essa dissertação apresenta.

Os resultados obtidos foram considerados satisfatórios, uma vez que o modelo mostrou a importância da sincronização do ritmo circadiano, do indivíduo ter uma boa noite de sono, sem dor, cumprindo com o objetivo proposto. Assim, o modelo apresenta um comportamento similar a realidade, podendo ser utilizado por pessoas que trabalham com a área médica.

\section{References}

Azevedo, L. L. (2007). AProSiMA - ambiente de resolução cooperativa de problemas baseado em simulação multiagentes. PhD thesis, Programa de Doutorado em Engenharia Elétrica, UFES, Vitória.

Borbely et al. (1984). Timing of human sleep: recovery process gated by a circadian pacemaker. American Journal of Physiology-Regulatory, Integrative and Comparative Physiology, 246(2):R161-R183.

Borbely, A. A. and Achermann, P. (1999). Sleep homeostasis and models of sleep regulation. Journal of biological rhythms, 14(6):559-570.

Chapman, C. R., Casey, K., Dubner, R., Foley, K., Gracely, R., and Reading, A. (1985). Pain measurement: an overview. Pain, 22(1):1-31.

Simoneau, J. L. et al. (1985). Human skeletal muscle fiber type alteration with highintensity intermittent training. European journal of applied physiology and occupational physiology, 54(3):250-253.

Skeldon, A. (2014). Are you listening to your body clock? http://personal. maths.surrey.ac.uk/st/A.Skeldon/sleep.html. [Online; accessed 20January -2018].

So, Y. and Durfee, E. H. (1996). Designing tree-structured organizations for computational agents. Computational \& Mathematical Organization Theory, 2(3):219-245. 